Pacific Journal of Mathematics

PRIMITIVE EXTENSIONS OF ARONSZAJN SPACES 


\title{
PRIMITIVE EXTENSIONS OF ARONSZAJN SPACES
}

\author{
Thomas M. Phillips
}

\begin{abstract}
The concept of a primitive representative of a primitive sequence is used to describe an internal property of a space which permits the construction of a model for a completion of an arbitrary completable Aronszajn space. It is shown that all the members of countable collection of completions of a given space contain a copy of a single completion of this form.
\end{abstract}

1. Introduction. A regular $T_{0}$ space having a base of countable order is called an Aronszajn space while a regular $T_{0}$ space having a $\lambda$-base (or equivalently a monotonically complete base of countable order) is called a complete Aronszajn space. Using the terminology in $\S 2.2$, it can be shown that a regular $T_{0}$ space is a (complete) Aronszajn space if and only if it has a (complete) Aronszajn sequence or equivalently a (complete) primitive Aronszajn sequence. An Aronszajn space $S$ which is homeomorphic to a dense subspace of complete Aronszajn space $X$ is said to be completable and $X$ is called a completion of $S$. (When convenient to do so, the words "homeomorphic to" are ignored in the usage of this definition.) The first example of a noncompletable Aronszajn space was given in [7; Theorem 8]. More recently, additional examples have been found as corollaries to other investigation. In particular, every Aronszajn space which does not contain a dense metrizable subspace (hence every nonseparable Aronszajn space in which there do not exist uncountably many mutually exclusive open sets) is noncompletable. Thus the spaces in [7; Theorem 1] and [8; Theorem 1] are also noncompletable Aronszajn spaces. The space $X$ constructed in [5; Theorem 3] and the space $\Lambda$ in [4] are other examples of particular interest since the former is separable and the latter is metacompact.

In this paper the so-called "primitive concepts" of Wicke and Worrell are used to describe an internal property of a space which permits the construction of a model for a completion of an arbitrary completable Aronszajn space. This work was motivated by the efforts of Alzoobaee [1], Green [2], Reed [6], and Whipple [9] to establish sufficient (and in some instances, necessary) conditions under which a Moore space has a completion or a semicompletion.

2. Preliminaries. It is assumed that the reader is familiar with the theory of primitive sequences and the related terminology found in [11]. The definitions presented in [11] will not be duplicated 
here. The following additional terminology and notation will be used throughout this paper.

Notation 2.1. $N$ denotes the set of positive integers and $(S, \tau)$ denotes a regular $T_{0}$ space. If $G$ is a sequence of collections of subsets of a set $X$, then $D(G)$ denotes the set of all decreasing representatives of $G$. If $G$ is a primitive sequence of $M$ in $X$, then $P(G)$ denotes the set of all primitive representatives of $G$. If $K$ is a well-ordered collection of sets and $u$ is a subset of some element of $K$, then $K(u)$ denotes the first element in $K$ which contains $u$. If $u=\{P\}$, then $K(P)$ denotes $K(u)$.

Terminology 2.2. (1) Suppose $f$ is a sequence of subsets of a space $X$. If $x$ is a point of $X$ every neighborhood of which contains a term of $f$, then $f$ is said to converge to $x$. A sequence $h$ of subsets of $X$ is coarser than $f$ (or $f$ is finer than $h$ ) provided every term of $h$ contains a term of $f$. If $f$ is finer than $h$ and coarser than $h$, then $f$ and $h$ are said to be equivalent. If $U \subseteq X$ and for each $n, f_{n} \cap U \neq \varnothing$, then $f$ is said to intersect $U$.

(2) If $\Gamma_{1}$ and $\Gamma_{2}$ are collections of sequences of sets, then $\Gamma_{1}$ is said to be finer than $\Gamma_{2}$ (or $\Gamma_{2}$ is coarser than $\Gamma_{1}$ ) if each element of $\Gamma_{1}$ is finer than some element of $\Gamma_{2}$. If $\Gamma_{1}$ is finer than $\Gamma_{2}$ and coarser than $\Gamma_{2}$, then $\Gamma_{1}$ and $\Gamma_{2}$ are said to be equivalent.

(3) If $G$ is a sequence of collections of sets and $\Gamma(G)$ is a collection of representatives of $G$, then a set $U$ is said to be $\Gamma(G)$-embedded in a set $V$ if $V$ contains a term of every element of $\Gamma(G)$ which intersects $U$.

(4) An Aronszajn sequence of $S$ is a decreasing sequence $G$ of bases for $S$ such that if $g \in D(G)$ and $P \in \bigcap_{n=1}^{\infty} g_{n}$, then $g$ converges to $P$. If each element of $D(G)$ converges in $S$, then $G$ is said to be complete.

(5) A primitive Aronszajn sequence of $S$ is a primitive closurewise sequence $W$ of $S$ such that if $w \in P(W)$ and $P \in \bigcap_{n=1}^{\infty} w_{n}$, then $w$ converges to $P$. If each element of $P(W)$ converges in $S$, then $W$ is said to be complete. $W$ is called completing if for each $w \in P(W)$ and $n \in N$, there is a $k \in N$ such that $w_{k}$ is $P(W)$-embedded in $w_{n}$. A complete primitive Aronszajn sequence of $S$ is clearly completing.

LEMMA 2.3. If $W$ and $V$ are primitive sequences of a subset $M$ of a set $X$, then $D(W)$ and $P(W)$ are equivalent and if $W_{n}(P) \subseteq$ $V_{n}(P)$ for each $P \in M$, then $P(W)$ is finer than $P(V)$.

Proof. These results follow from the definitions and the results in $[10]$. 
LEMma 2.4. If $S$ is a subspace of a regular space $X$ and $G$ (resp. B) is a sequence of bases for $X$ (resp. S), then there is a primitive closurewise sequence $W$ of $S$ such that $W_{n} \subseteq B_{n}, P(W)$ is finer than $D(G)$, and for each $w \in \bigcup_{n=1}^{\infty} W_{n}$, there is an open set $U(w)$ in $X$ such that $U(w) \cap S=w$ and if $P \in S$ and $n \in N$, then

$$
\overline{U\left(W_{n+1}(P)\right)^{X}} \subseteq U\left(W_{n}(P)\right) .
$$

Proof. By the proof of [10; Lemma 2.1], there is a primitive closurewise sequence $H$ of $S$ in $X$ such that $H_{n} \subseteq G_{n}$. For each $n$, let $B_{n}$ be well-ordered. Let $V_{1}=\left\{b \in B_{1} \mid b\right.$ is contained in some set in $\left.H_{1}\right\}$ be well-ordered so that $x \leqq y$ in $V_{1}$ if and only if (1) $H_{1}(x)<H_{1}(y)$ or $(2) H_{1}(x)=H_{1}(y)$ and $x \leqq y$ in $B_{1}$. Then $W_{1}=$ $\left\{V_{1}(P) \mid P \in S\right\}$ covers $S$ and $W_{1}(P)=V_{1}(P)$ for each $P \in S$. Let $P \in S$. Clearly $H_{1}(P) \leqq H_{1}\left(W_{1}(P)\right)$. Suppose $H_{1}(P)<H_{1}\left(W_{1}(P)\right)$. There is a set $b$ in $B_{1}$ such that $P \in b$ and $b \subseteq H_{1}(P)$. Thus $b \in V_{1}$ and $H_{1}(b)=$ $H_{1}(P)<H_{1}\left(W_{1}(P)\right)$; hence $V_{1}(P) \leqq b<W_{1}(P)$. This is a contradiction. Thus $H_{1}(P)=H_{1}\left(W_{1}(P)\right)$ and $W_{1}(P) \subseteq H_{1}(P)$. For each $w \in W_{1}$, let $U(w)$ be an open set in $X$ such that $U(w) \cap S=w$.

If $A \subseteq S$, then $\bar{A}^{x}$ denotes the closure of $A$ in $X$ and $\bar{A}$ denotes the closure of $A$ in $S$. Let $V_{2}=\left\{b \in B_{2} \mid \bar{b}\right.$ is contained in some set in $H_{2}$ and some set in $W_{1}$ and there is an open set $u$ in $X$ such that $u^{-x} \subseteq U\left(W_{1}(b)\right)$ and $\left.u \cap S=b\right\}$ be well-ordered so that $x \leqq y$ in $V_{2}$ if and only if

(1) $H_{2}(\bar{x})<H_{2}(\bar{y})$; or

(2) $H_{2}(\bar{x})=H_{2}(\bar{y})$ and $W_{1}(\bar{x})<W_{1}(\bar{y})$; or

(3) $H_{2}(\bar{x})=H_{2}(\bar{y})$ and $W_{1}(\bar{x})=W_{1}(\bar{y})$ and $x \leqq y$ in $B_{2}$. Then $W_{2}=\left\{V_{2}(P) \mid P \in S\right\}$ covers $S$ and $W_{2}(P)=V_{2}(P)$ for each $P \in S$. Let $P \in S$. There exists a set $b$ in $B_{2}$ and an open set $u$ in $X$ such that $P \in b=u \cap S, u^{-X} \subseteq U\left(W_{1}(P)\right)$, and $\bar{b} \subseteq W_{1}(P) \cap H_{2}(P)$. Since $W_{1}(P)=W_{1}(b), b$ is in $V_{2}$. An argument like the one in the preceding paragraph will show that $H_{2}(P)=H_{2}\left(\overline{W_{2}(P)}\right)$; hence $W_{2}(P) \subseteq H_{2}(P)$. Clearly $W_{1}(P) \leqq W_{1}\left(\overline{W_{2}(P)}\right)$. If $W_{1}(P)<W_{1}\left(\overline{W_{2}(P)}\right)$, then $H_{2}(\bar{b})=H_{2}(P)=$ $H_{2}\left(\overline{W_{2}(P)}\right)$ and $W_{1}(\bar{b})=W_{1}(P)<W_{1}\left(\overline{W_{2}(P)}\right)$ imply that $V_{2}(P) \leqq b<$ $W_{2}(P)$ which is a contradiction. So $W_{1}(P)=W_{1}\left(\overline{W_{2}(P)}\right)$ and $\left(\overline{W_{2}(P)}\right) \subseteq$ $W_{1}(P)$. By definition of $V_{2}$, there is an open set $U\left(W_{2}(P)\right)$ in $X$ such that $\overline{U\left(W_{2}(P)\right)^{X}} \subseteq U\left(W_{1}\left(W_{2}(P)\right)\right)=U\left(W_{1}(P)\right)$ and $U\left(W_{2}(P)\right) \cap S=W_{2}(P)$.

This process may be continued to construct a primitive closurewise sequence $W$ of $S$ such that $W_{n} \subseteq B_{n}, W_{n}(P) \subseteq H_{n}(P)$, and the desired property for $U(w)$ holds. As in Lemma 2.3, $P(W)$ is finer than $D(G)$.

LEMMA 2.5. If $H$ is an open primitive sequence of $S$ and for each $n \in N, G^{n}=\left(G_{m}^{n}\right)_{m=1}^{\infty}$ is a primitive Aronszajn sequence of $S$ such that if $U$ is a neighborhood of $P \in S$ and $n \in N$, there is a neighbor- 
hood of $P$ which is $P\left(G^{n}\right)$-embedded in $U$, then there is a completing primitive Aronszajn sequence $W$ of $S$ such that $P(W)$ is finer than $P(H)$ and for each $n, P(W)$ is finer than $P\left(G^{n}\right)$ and if $m \geqq n$, then $W_{m+1}(P)$ is $P\left(G^{n}\right)$ - embedded in $W_{m}(P)$ for each $P \in S$.

Proof. Let $\tau$ be well-ordered and let $V_{1}=\{u \in \tau \mid u$ is contained in some set in $G_{1}^{1}$ and some set in $\left.H_{1}\right\}$ be well-ordered so that $x \leqq y$ in $V_{1}$ if and only if

(1) $G_{1}^{1}(x)<G_{1}^{1}(y)$; or

(2) $G_{1}^{1}(x)=G_{1}^{1}(y)$ and $H_{1}(x)<H_{1}(y)$; or

(3) $G_{1}^{1}(x)=G_{1}^{1}(y)$ and $H_{1}(x)=H_{1}(y)$ and $x \leqq y$ in $\tau$.

An argument similar to that in the proof of Lemma 2.4 will show that $W_{1}=\left\{V_{1}(P) \mid P \in S\right\}$ covers $S$ and if $P \in S$, then $G_{1}^{1}(P)=G_{1}^{1}\left(W_{1}(P)\right)$ and $H_{1}(P)=H_{1}\left(W_{1}(P)\right)$ so that $W_{1}(P) \subseteq G_{1}^{1}(P) \cap H_{1}(P)$.

Suppose $k \in N$ and $W_{1}, \cdots, W_{k}$ are well-ordered open covers of $S$ such that if $n \leqq k$, then

$\left(I_{1}\right)$ if $w \in W_{n}$, there is a point $P$ such that $w=W_{n}(P)$;

$\left(I_{2}\right) \quad W_{n}(P) \subseteq H_{n}(P) \cap \bigcap_{i=1}^{n} G_{n}^{i}(P)$;

and if $k>1$ and $n \leqq k-1$, then

$\left(I_{3}\right) \quad W_{n+1}(P)$ is $P\left(G^{i}\right)$-embedded in $W_{n}(P)$ for $i=1, \cdots, n$.

Let $V_{k+1}=\left\{u \in \tau \mid u\right.$ is contained in some set in each of $H_{k+1}, G_{k+1}^{1}$, $\cdots, G_{k+1}^{k+1}$ and there is a set $v$ in $W_{k}$ such that $u$ is $P\left(G^{i}\right)$-embedded in $v$ for $i=1, \cdots, k\}$ be well-ordered so that $x \leqq y$ in $V_{k+1}$ if and only if

$\left(1^{\prime}\right)$ there is an integer $i \leqq k+1$ such that $G_{k+1}^{i}(x) \neq G_{k+1}^{i}(y)$ and if $j$ is the least such integer, then $G_{k+1}^{j}(x)<G_{k+1}^{j}(y)$; or

(2') $G_{k+1}^{i}(x)=G_{k+1}^{i}(y)$ for $i=1, \cdots, k+1$ and

(a) $H_{k+1}(x)<H_{k+1}(y)$; or

(b) $H_{k+1}(x)=H_{k+1}(y)$ and the first set $u$ in $W_{k}$ in which $x$ is $P\left(G^{i}\right)$-embedded for $i=1, \cdots, k$ strictly precedes the first set $v$ in $W_{k}$ in which $y$ is $P\left(G^{i}\right)$-embedded for $i=1, \cdots, k$; or

(c) $H_{k+1}(x)=H_{k+1}(y)$ and $u=v$ and $x \leqq y$ in $\tau$.

Then $W_{k+1}=\left\{V_{k+1}(P) \mid P \in S\right\}$ covers $S$ and $W_{k+1}(P)=V_{k+1}(P)$ for each $P \in S$. Thus $\left(I_{1}\right)$ holds for $n=k+1$. Let $P \in S$. It again follows as in the proof of Lemma 2.4 that $H_{k+1}(P)=H_{k+1}\left(W_{k+1}(P)\right)$ and $G_{k+1}^{i}(P)=G_{k+1}^{i}\left(W_{k+1}(P)\right)$ for $i=1, \cdots, k+1$. Thus $\left(I_{2}\right)$ holds for $n=k+1$. There is a set $u$ in $V_{k+1}$ such that $P \in u \subseteq H_{k+1}(P) \cap \bigcap_{i=1}^{k+1} G_{k+1}^{i}(P)$ and $u$ is $P\left(G^{i}\right)$ embedded in $W_{k}(P)$ for $i=1, \cdots, k$. Let $w$ be the first set in $W_{k}$ in which $W_{k+1}(P)$ is $P\left(G^{i}\right)$-embedded for $i=1, \cdots, k$. Then $W_{k}(P) \leqq$ $w$. Note that $G_{k+1}^{i}(u)=G_{k+1}^{i}(P)=G_{k+1}^{i}\left(W_{k+1}(P)\right)$ for $i=1, \cdots, k+1$ and $H_{k+1}(u)=H_{k+1}(P)=H_{k+1}\left(W_{k+1}(P)\right)$. Also note that the first set in $W_{k}$ in which $u$ is $P\left(G^{i}\right)$-embedded for $i=1, \cdots, k$ is not preceded by $W_{k}(P)$. Thus if $W_{k}(P)<w$, then $V_{k+1}(P) \leqq u<W_{k+1}(P)$ which is 
a contradiction. So $W_{k}(P)=w$. Hence $W_{k+1}(P)$ is $P\left(G^{i}\right)$-embedded in $W_{k}(P)$ for $i=1, \cdots, k$ and $\left(I_{3}\right)$ holds for $n=k$.

It follows that there is a sequence $W$ of well-ordered open covers of $S$ such that $\left(I_{1}\right)-\left(I_{3}\right)$ hold for all $n$. By $\left(I_{3}\right), \overline{W_{n+1}(P)} \subseteq W_{n}(P)$ which, along with $\left(I_{1}\right)$, implies that $W$ is a primitive closurewise sequence of $S$. By Lemma 2.3, $P(W)$ is finer than $P(H)$ since $W_{n}(P) \subseteq H_{n}(P)$ by $\left(I_{2}\right)$. Similarly it can be shown that $W_{n}(P) \subseteq G_{n}^{i}(P)$ for $i \leqq n$ implies that $P(W)$ is finer than $P\left(G^{i}\right)$ for each $i \in N$. The fact that $P(W)$ is finer than $P\left(G^{1}\right)$ implies that $W$ is a primitive Aronszajn sequence of $S$ and, along with $\left(I_{3}\right)$, that $W_{n+1}(P)$ is $P(W)$-embedded in $W_{n}(P)$. Thus $W$ is completing.

\section{Primitive extensions of Aronszajn spaces.}

Definition 3.1. Suppose $S$ has a primitive Aronszajn sequence $W$ and for each $x \in P(W)$, let $[x]=\{y \in P(W) \mid x$ and $y$ are equivalent $\}$. For each $U \in \tau$, let $U^{*}=\{[x] \mid U$ contains a term of $x\}$. Then $\left\{U^{*} \mid U \in \tau\right\}$ is a base for a topology $\tau^{*}$ on $S^{*}$ and $S$ is homeomorphic to a dense subspace of $S^{*}$. The space $\left(S^{*}, \tau^{*}\right)$ will be denoted by $S_{P}^{*}(W)$ and will be called the primitive extension of $S$ by $W$.

THEOREM 3.2. If $W$ is a primitive Aronszajn sequence of $S$, then $S_{P}^{*}(W)$ is a $T_{0}$ space having a complete primitive base [12]. Hence if $S_{P}^{*}(W)$ is regular, then it is a complete Aronszajn space.

Proof. For each $n$, let $W_{n}^{*}=\left\{w^{*} \mid w \in W_{n}\right\}$ be well-ordered so that $U<V$ in $W_{n !}^{*}$ if and only if the first element $u$ of $W_{n}$ such that $u^{*}=U$ strictly precedes the first element $v$ of $W_{n}$ such that $v^{*}=V$. Then $W^{*}=W_{1}^{*}, W_{2}^{*}, \cdots$ has the property in the definition of a complete primitive base given in [12]. The second conclusion follows from [12; Theorem 3].

THEOREM 3.3. If $W$ is a primitive Aronszajn sequence of $S$, then $S_{P}^{*}(W)$ is a complete Aronszajn space if and only if $W$ is completing.

Proof. The property in the definition of a completing primitive Aronszajn sequence of $S$ is easily shown to be a necessary and sufficient condition for $S_{P}^{*}(W)$ to be regular. The result then follows.

THEOREM 3.4. $S$ is a completable Aronszajn space if and only if $S$ has a completing primitive Aronszajn sequence.

Proof. Suppose $S$ is a completable Aronszajn space and let $X$ 
be a complete Aronszajn space containing $S$. Let $B$ (resp. $G$ ) be a (complete) Aronszajn sequence of $S$ (resp. $X$ ) and let $W$ be as in Lemma 2.4. Since $W_{n} \subseteq B_{n}$, it follows that $W$ is a primitive Aronszajn sequence of $S$. Let $P \in S$ and $n \in N$ and suppose $w \in P(W)$ intersects $W_{n+1}(P)$. Let $g$ be an element of $D(G)$ which is coarser than $w$. Since $G$ is complete, $g$ converges to some point $Q \in X$ and $\left.Q \in \overline{W_{n+1}(P}\right)^{X} \subseteq$ $\overline{U\left(W_{n+1}(P)\right)^{X}} \cong U\left(W_{n}(P)\right)$. So $U\left(W_{n}(P)\right)$ contains a term of $g$ and hence $W_{n}(P)$ contains a term of $w$. Thus $W_{n+1}(P)$ is $P(W)$-embedded in $W_{n}(P)$ and $W$ is completing.

The converse follows from Theorem 3.3.

Corollary 3.5. $S$ is a completable Aronszajn space if and only if there is an Aronszajn sequence $B$ of $S$ such that if $P \in S$, then every neighborhood of $P$ contains a $D(B)$-embedded neighborhood of $P$.

Proof. Suppose $S$ is a completable Aronszajn space and let $W$ be a completing primitive Aronszajn sequence of $S$. For each $n$, let $B_{n}=\bigcup_{i \geqq n} W_{i}$. Then $B=B_{1}, B_{2}, \ldots$ is a decreasing sequence of bases for $S$ and by [10; Lemma 2.2], $D(B)$ is finer than $P(W)$. This latter property implies that $B$ is an Aronszajn sequence of $S$. Let $P \in S$ and let $U$ be a neighborhood of $P$. There are integers $n$ and $k$ such that $W_{k}(P)$ is $P(W)$-embedded in $W_{n}(P)$ and $W_{n}(P) \subseteq U$. Since $D(B)$ is finer than $P(W), W_{k}(P)$ is $D(B)$-embedded in $U$.

Conversely if $B$ is a sequence as described in the corollary and $G$ is a primitive closurewise sequence of $S$ such that $G_{n} \subseteq B_{n}$, then $G$ has the property of $G^{n}$ in Lemma 2.5 and by that lemma, $S$ has a completing primitive Aronszajn sequence.

REMARK 3.6. By Theorems 3.3 and 3.4, if $S$ is a completable Aronszajn space, then $S$ has a completion of the form $S_{P}^{*}(W)$ for some completing primitive Aronszajn sequence $W$ of $S$. The example given below shows that not all completions of $S$ need be of this form. However, by Theorem 3.8, every completion of $S$ contains a completion of this form.

ExAmple 3.7. There exists a completable Aronszajn space $S$ and a completion $X$ of $S$ such that $X$ is not homeomorphic to $S_{P}^{*}(W)$ for any primitive Aronszajn sequence $W$ of $S$.

Proof. Let $Q$ (the space of all rational numbers), $R$ (the space of all real numbers) and $N$ have their usual topologies and let $S=$ $N \times Q$ have the product topology. Let $B$ be a collection of increasing sequences of positive integers which is maximal with respect to the property that if $x$ and $y$ are distinct elements of $B$, then $\left\{n \mid x_{n}=y_{n}\right\}$ 
is at most finite. $B$ is uncountable. Let $X=B \cup(N \times R)$ be topologized so that $N \times R$ has the product topology and for each $x \in B$, basic open sets about $x$ are of the form $U(x ; n)=\{x\} \cup\left\{\left(x_{k}, t\right) \mid k \geqq n\right.$ and $t \in R\}$ for each $n \in N . \quad X$ is a complete Aronszajn space having $S$ as a dense subspace. If $W$ is a primitive Aronszajn sequence of $S$, then $W_{n}$ is countable for each $n$; hence $\left\{w^{*} \mid w \in \bigcup_{n=1}^{\infty} W_{n}\right\}$ is a countable base for $S_{P}^{*}(W)$. Since $X$ is not metrizable, $X$ cannot be homeomorphic to $S_{P}^{*}(W)$.

THEOREM 3.8. If $S$ is a completable Aronszajn space and $X$ is a completion of $S$, then there is a completing primitive Aronszajn sequence $W$ of $S$ such that $S_{P}^{*}(W)$ is homeomorphic to a subspace of $X$.

Proof. We will assume that $S$ is a subspace of $X$. Let $B$ (resp. $G$ ) be a (complete) Aronszajn sequence of $S$ (resp. $X$ ) and let $W$ be as in Lemma 2.4. As in the proof of Theorem 3.4, $W$ is a completing primitive Aronszajn sequence of $S$. Since every element of $P(W)$ converges in $X$, the mapping $\phi$ from $S_{P}^{*}(W)$ into $X$ given by $\phi([x])=$ $\lim x$ is well-defined.

Suppose $x$ and $y$ are elements of $P(W)$ which converge to the same point $P$ in $X$. Let $n \in N$. Using the notation of Lemma 2.4, we have that $P \in \bar{y}_{n+1}^{X} \subseteq \overline{U\left(y_{n+1}\right)^{X}} \subseteq U\left(y_{n}\right)$. So $U\left(y_{n}\right)$, hence $y_{n}$, contains a term of $x$. Thus $x$ is finer than $y$ and similarly $y$ is finer than $x$. Hence $x$ and $y$ are equivalent and $\phi$ is an injection.

Let $w \in P(W)$ and let $U$ and $V$ be open sets in $X$ such that $\dot{\phi}([w]) \in V$ and $\bar{V}^{x} \subseteq U$. There is an $n$ such that $w_{n} \subseteq V$. If $[z] \in w_{n}^{*}$, then $z_{j} \leqq w_{n}$ for some $j$ hence $\phi([z]) \in \bar{z}_{j}^{X} \cong \bar{w}_{n}^{X} \cong \bar{V}^{X} \cong U$. So $\dot{\phi}$ is continuous at $[w]$.

Suppose $u \in \tau$ and $[x] \in u^{*}$. For some $n, x_{n} \leqq u$ and $\phi([x])=\lim x \in$ $\bar{x}_{n+1}^{X} \subseteq \overline{U\left(x_{n+1}\right)^{X}} \leqq U\left(x_{n}\right)$. If $y \in P(W)$ and $\phi([y])=\lim y \in U\left(x_{n}\right)$, then $U\left(x_{n}\right)$, hence $x_{n}$, contains a term of $y$. Thus $[y] \in x_{n}^{*} \subseteq u^{*}$ and it follows that $\phi\left(u^{*}\right)$ is open in $\phi\left(S_{P}^{*}(W)\right)$. So $\phi$ is a homeomorphism.

COROLlary 3.9. If $S$ is a completable Aronszajn space and $X_{1}, X_{2}, \cdots$ are completions of $S$, then there is a completing primitive Aronszajn sequence $W$ of $S$ such that $S_{P}^{*}(W)$ is homeomorphic to a subspace of $X_{n}$ for each $n$.

Proof. By Theorem 3.8, there is a completing primitive Aronszajn sequence $G^{n}$ of $S$ such that $S_{P}^{*}\left(G^{n}\right)$ is homeomorphic to a subspace of $X_{n}$. By Lemma 2.5, there is a completing primitive Aronszajn sequence $W$ of $S$ such that for each $n, P(W)$ is finer than $P\left(G^{n}\right)$ and for $m \geqq n, W_{m+1}(P)$ is $P\left(G^{n}\right)$-embedded in $W_{m}(P)$ for each $P \in S$.

Let $n \in N$ and let $\phi$ be the mapping from $S_{P}^{*}(W)$ into $S_{P}^{*}\left(G^{n}\right)$ such 
that if $w \in P(W)$, then $\dot{\phi}([w])=[g]$ where $g$ is any element of $P\left(G^{n}\right)$ which is coarser than $w$. Since $w_{m+1}$ is $P\left(G^{n}\right)$-embedded in $w_{m}$ for $m \geqq n$, it follows that $w$ and $g$ are equivalent. Hence $\phi$ is well-defined and injective. It is easy to verify that $\phi$ is a homeomorphism of $S_{P}^{*}(W)$ into $S_{P}^{*}\left(G^{n}\right)$ and hence into $X_{n}$.

COROLlaRY 3.10. If $P$ is a hereditary topological property in the class of Aronszajn spaces and $S$ is a completable Aronszajn space some completion of which has property $P$, then every completion of $S$ contains a completion of $S$ having property $P$.

Question 3.11. For what topological properties $P$ is it true that if $S$ is a completable Aronszajn space having property $P$, then $S$ has a completion having property $P$ ?

THEOREM 3.12. If $S$ is a completable Aronszajn space having either a point-countable base or a $\sigma$-disjoint base, then $S$ has a completion having such a base.

Proof. Let $A$ be a base for $S$. There is an Aronszajn sequence $B$ of $S$ such that $B_{n} \leqq A$ for each $n$. As in the proof of Theorem 3.4, there is a completing primitive Aronszajn sequence $W$ of $S$ such that $W_{n} \subseteq B_{n}$ for each $n$. Then $\left\{w^{*} \mid w \in \bigcup_{n=1}^{\infty} W_{n}\right\}$ is a base for $S_{P}^{*}(W)$ which is easily shown to be point-countable or $\sigma$-disjoint according as $A$ is point-countable or $\sigma$-disjoint.

TheOREM 3.13. If $S$ is a locally connected completable Aronszajn space, then $S$ has a locally connected completion.

Proof. Let $A$ be a base for $S$ consisting of connected sets and let $W$ be as in the proof of Theorem 3.12. Let $\theta$ be the natural embedding of $S$ into $S_{P}^{*}(W)$. For each $w \in \bigcup_{n=1}^{\infty} W_{n}, \theta(w)$ is a dense connected subset of $w^{*}$ so $w^{*}$ is connected. Hence $\left\{w^{*} \mid w \in \bigcup_{n=1}^{\infty} W_{n}\right\}$ is a base for $S_{P}^{*}(W)$ consisting of connected sets.

Theorem 3.14. If $S$ is a completable Aronszajn space and $\phi$ is a continuous mapping of $S$ into a complete Aronszajn space $X$, then $\phi$ has a continuous extension to a completion of $S$.

Proof. The techniques in [10] can be used to show that there is an Aronszajn sequence $Z$ of $\phi(S)$ such that every element of $D(Z)$ converges in $X$. For each $n$, let $\phi^{-1}\left(Z_{n}\right)=\left\{\phi^{-1}(z) \mid z \in Z_{n}\right\}$. Then $\left(\phi^{-1}\left(Z_{n}\right)\right)_{n=1}^{\infty}$ is an open monotonically contracting sequence of $S$ and by [10; Lemma 2.1] there is a primitive sequence $H$ of $S$ such that 
$H_{n} \subseteq \phi^{-1}\left(Z_{n}\right)$ for each $n$. Let $G$ be a completing primitive Aronszajn sequence of $S$. By Lemma 2.5, there is a completing primitive Aronszajn sequence $W$ of $S$ such that $P(W)$ is finer than $P(H)$. If $w \in P(W)$ and $h \in P(H)$ is coarser than $w$, then $\phi(h) \in D(Z)$ and $\phi(h)$, hence $\phi(w)$, converges in $X$. So the mapping $\psi$ from $S_{P}^{*}(W)$ into $X$ defined by $\psi([w])=\lim \phi(w)$ is continuous and if $\theta$ is the natural embedding of $S$ into $S_{P}^{*}(W)$, then $\psi(\theta(x))=\phi(x)$ for each $x \in S$. The result follows.

4. Moore spaces. A regular $T_{0}$ developable space is called a Moore space. Every Moore space is an Aronszajn space and a Moore space which is a complete Aronszajn space is said to be semicomplete. Using the terminology in $\S 4.1$, a regular $T_{0}$ space is a (semicomplete) Moore space if and only if it has a (semicomplete) decreasing development or equivalently a (semicomplete) primitive development. The term "complete" in the context of Moore spaces is reserved for spaces satisfying Moore's Axiom 1 [3]. The space described in [7; Theorem 9] is a semicomplete Moore space which is not complete. A Moore space which is homeomorphic to a dense subspace of a semicomplete Moore space is called semicompletable. All of the examples of noncompletable Aronszajn spaces cited in the introduction are nonsemicompletable Moore spaces.

DEFINITION 4.1. If $G$ is a sequence of collections of sets and $\Gamma(G)$ is a collection of representatives of $G$, then a set $U$ is said to be uniformly $\Gamma(G)$-embedded in $V$ if for each $g \in \Gamma(G)$ which intersects $U$, there is an $n \in N$ such that $V$ contains every set in $G_{n}$ which is intersected by $g$. A sequence $G$ of open covers of a space $X$ having the property that if $g$ is a representative of $G$ and $x \in \bigcap_{n=1}^{\infty} g_{n}$, then $g$ converges to $x$ is called a development of $X$ and $X$ is said to be developable. A development $G$ of $X$ is called semicomplete if each element of $D(G)$ converges in $X$. A (semicomplete) primitive development of $X$ is a (semicomplete) development of $X$ which is a primitive closurewise sequence of $X$. A primitive development $W$ of $X$ is said to be semicompleting if for each $w \in P(W)$ and $n \in N$, there is a $k \in N$ such that $w_{k}$ is uniformly $P(W)$-embedded in $w_{n}$.

THEOREM 4.2. If $W$ is a semicompleting primitive development of $S$, then $S_{P}^{*}(W)$ is a semicomplete Moore space.

REMARK 4.3. Results and questions analogous to those in paragraphs 3.4 through 3.14 of the previous section can be obtained for semicompletable Moore spaces by the proper translation of terminology. The term "Aronszajn sequence" without the adjective "primitive" 
must be replaced by the term "decreasing development." In some instances, the proofs require obvious modifications.

\section{REFERENCES}

1. O. H. Alzoobaee, Completion of Moore spaces, Thesis, University of Iowa, 1962.

2. J. W. Green, Completion and semicompletion of Moore spaces, Pacific J. Math., 57 (1975), 153-165.

3. R. L. Moore, Foundation of Point Set Theory, Amer. Math. Soc. Colloq. Pub., Vol. 13, rev. ed., 1962.

4. C. Pixley and P. Roy, Uncompletable Moore spaces, Proc. Top. Conf. Auburn University, (1969), 75-85.

5. G. M. Reed, Concerning completable Moore spaces, Proc. Amer. Math. Soc., 36 (1972), 591-596.

6. J. Reed, On completeness and semicompleteness of first countable spaces, Pacific J. Math., 55 (1974), 553-563.

7. M. E. Rudin, Concerning abstract spaces, Duke Math. J., 17 (1950), 317-327.

8. - Separation in nonseparable spaces, Duke Math. J., 18 (1951), 623-629.

9. K. E. Whipple, Cauchy sequences in Moore spaces, Pacific J. Math., 18 (1966), 191-199.

10. H. H. Wicke and J. M. Worrell Jr., Topological completeness in first countable Hausdorff spaces I, Fund. Math., 75 (1972), 209-222.

11. - Primitive structures in general topology, Proc. Top. Conf. University of North Carolina at Charlotte, Academic Press, New York, 1974, 581-599.

12. J. M. Worrell Jr. and H. H. Wicke, A central metrization theorem II, Notices Amer. Math. Soc., 20 (1973), A-381.

Received May 25, 1977 and in revised form September 27, 1977.

AUBURN UNIVERSITY

AUbuRn, AL 36830 


\section{PACIFIC JOURNAL OF MATHEMATICS}

\section{EDITORS}

RICHARD ARENS (Managing Editor)

University of California

Los Angeles, California 90024

C. W. Curtis

University of Oregon

Eugene, OR 97403

C. C. MOORE

University of California

Berkeley, CA 94720

\section{J. DUGUNDJI}

Department of Mathematics University of Southern California Los Angeles, California 90007

R. Finn AND J. Milgram Stanford University Stanford, California 94305

\section{ASSOCIATE EDITORS}

E. F. BeCK ENBACH

B. H. NeUMaNN

F. WOLF

K. Yoshida

\section{SUPPORTING INSTITUTIONS}

UNIVERSITY OF BRITISH COLUMBIA CALIFORNIA INSTITUTE OF TECHNOLOGY UNIVERSITY OF CALIFORNIA MONTANA STATE UNIVERSITY UNIVERSITY OF NEVADA, RENO NEW MEXICO STATE UNIVERSITY OREGON STATE UNIVERSITY UNIVERSITY OF OREGON
UNIVERSITY OF SOUTHERN CALIFORNIA STANFORD UNIVERSITY UNIVERSITY OF HAWAII UNIVERSITY OF TOKYO UNIVERSITY OF UTAH WASHINGTON STATE UNIVERSITY UNIVERSITY OF WASHINGTON 


\section{Pacific Journal of Mathematics \\ Vol. 77, No. $1 \quad$ January, 1978}

Dan Amir, Chebyshev centers and uniform convexity ............... 1

Lawrence Wasson Baggett, Representations of the Mautner group. I ..... 7

George Benke, Trigonometric approximation theory in compact totally

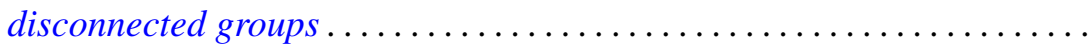

M. Bianchini, O. W. Paques and M. C. Zaine, On the strong compact-ported topology for spaces of holomorphic mappings ..................

Marilyn Breen, Sets with $(d-2)$-dimensional kernels

J. L. Brenner and Allen Kenneth Charnow, Free semigroups of $2 \times 2$

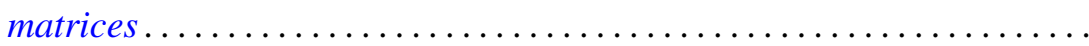

David Bressoud, A new family of partition identities .................

David Fleming Dawson, Summability of matrix transforms of stretchings

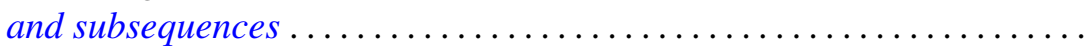

Harold George Diamond and Paul Erdôs, A measure of the nonmonotonicity

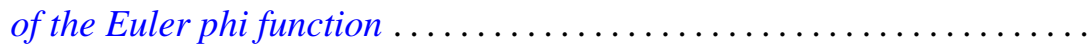

Gary Doyle Faulkner and Ronald Wesley Shonkwiler, Kernel dilation in reproducing kernel Hilbert space and its application to moment

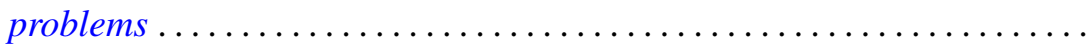

Jan Maksymilian Gronski, Classification of closed sets of attainability in the

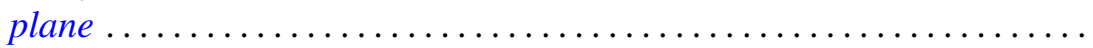

H. B. Hamilton and T. E. Nordahl, Semigroups whose lattice of congruences

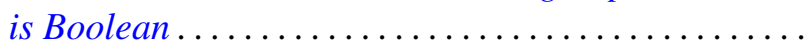

Harvey Bayard Keynes and D. Newton, Minimal $(G, \tau)$-extensions ...

Anthony To-Ming Lau, The Fourier-Stieltjes algebra of a topological

semigroup with involution.

B. C. Oltikar and Luis Ribes, On prosupersolvable groups ...

Brian Lee Peterson, Extensions of pro-affine algebraic groups ...

Thomas M. Phillips, Primitive extensions of Aronszajn spaces ...

Mehdi Radjabalipour, Equivalence of decomposable and 2-decomposable operators. .

M. Satyanarayana, Naturally totally ordered semigroups .

Fred Rex Sinal, A homeomorphism classification of wildly imbedded two-spheres in $S^{3}$

Hugh C. Williams, Some properties of a special set of recurring

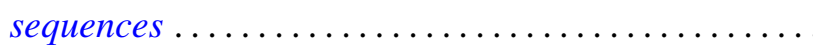

